\title{
Discerning the Antimicrobial Resistance, Virulence, and Phylogenetic Relatedness of Salmonella Isolates Across the Human, Poultry, and Food Materials Sources in Malaysia
}

OPEN ACCESS

Edited by:

Renee Lay Hong Lim

UCSI University, Malaysia

Reviewed by:

Min Yue,

Zhejiang University, China Xiaodong Xia,

Northwest A\&F University, China Zhiming Pan,

Yangzhou University, China

*Correspondence:

Zunita Zakaria

zunita@upm.edu.my

Specialty section:

This article was submitted to Antimicrobials, Resistance

and Chemotherapy,

a section of the journal

Frontiers in Microbiology

Received: 12 January 2021 Accepted: 09 August 2021

Published: 31 August 2021

Citation:

Zakaria Z, Hassan L, Ahmad N, Husin SA, Ali RM, Sharif Z, Sohaimi NM and Garba B (2021)

Discerning the Antimicrobial Resistance, Virulence,

and Phylogenetic Relatedness of Salmonella Isolates Across the Human, Poultry, and Food

Materials Sources in Malaysia.

Front. Microbiol. 12:652642. doi: 10.3389/fmicb.2021.652642

\begin{abstract}
Zunita Zakaria1,2*, Latiffah Hassan²,3, Norazah Ahmad4, Suraya Amir Husin, Rohaya Mohd Ali ${ }^{6}$, Zawiyah Sharif7, Norfitriah Mohamed Sohaimi ${ }^{3}$ and Bashiru Garba ${ }^{2,8}$

${ }^{1}$ Institute of Bioscience, Universiti Putra Malaysia, Serdang, Malaysia, ${ }^{2}$ Department of Veterinary Pathology and Microbiology, Faculty of Veterinary Medicine, Universiti Putra Malaysia, Serdang, Malaysia, ${ }^{3}$ Department of Veterinary Laboratory Diagnostics, Faculty of Veterinary Medicine, Universiti Putra Malaysia, Serdang, Malaysia, ${ }^{4}$ Infectious Diseases Research Centre, Institute for Medical Research, National Institutes of Health, Selangor, Malaysia, ${ }^{5}$ Medical Development Division, Ministry of Health, Putrajaya, Malaysia, ${ }^{6}$ Diagnostic and Quality Assurance Division, Department of Veterinary Services, Ministry of Agriculture and Agro-Based Industry, Putrajaya, Malaysia, ${ }^{7}$ Food Safety and Quality Division, Ministry of Health, Selangor, Malaysia, ${ }^{8}$ Department of Veterinary Public Health and Preventive Medicine, Faculty of Veterinary Medicine, Usmanu Danfodiyo University, Sokoto, Nigeria
\end{abstract}

Salmonella enterica subspecies enterica serovar Enteritidis is one of the major foodborne zoonotic pathogens globally. It has significantly impacted human health and global trade. In this investigation, whole-genome sequencing was employed to determine the antimicrobial resistance (AMR) pattern of a collection of Salmonella Enteritidis isolated from humans, poultry, and food sources. The study also investigated the virulence genes profile of the isolates as well as the phylogenetic relationships among strains. Illumina NextSeq technology was used to sequence the genome of 82 Salmonella Enteritidis strains isolated over 3 years (2016-2018) in Peninsular Malaysia. The pattern of resistance showed that tetracycline had the highest frequency (37/82, $45.12 \%)$, and isolates from food samples showed the highest rate of $9 / 18(50.00 \%)$, followed by human 17/35 (48.57\%) and then poultry 11/29 (37.93\%). The second drug with the highest resistance rate is ampicillin with 5/29 (17.24\%) for poultry, 4/35 (11.43\%) for human, and 0/18 (0.00\%) for food isolates respectively. Similarly, a total of 19 antimicrobial resistance (AMR) genes corresponding to the nine drugs used in the disc diffusion assay were evaluated from the whole genome sequence data. The aminoglycoside resistance gene aac $\left(6^{\prime}\right)$-ly was detected in 79 of the 82 isolates (96.34\%). While the phylogenetic analysis revealed distinct lineages isolated, the three sources indicating possible cross-contamination. In conclusion, the results showed that the genomic profile of Salmonella Enteritidis isolated from humans, poultry, and food samples share genetic traits, hence the need to institute measures at controlling the continuous spread of these resistant pathogens.

Keywords: Salmonella, whole-genome sequencing, antimicrobial resistance, phylogenetic studies, virulence gene profile, foodborne infections, Salmonella enteritidis 


\section{INTRODUCTION}

Salmonella is a very important zoonotic pathogen that has been reported to cause over 200 million human clinical infections with an estimated mortality of 3 million annually (Coburn et al., 2007; Graham et al., 2018; Moussa et al., 2021). Humans easily acquire infection from contact with animal or environmental reservoirs. The emergence of antimicrobial-resistant Salmonella enterica subspecies enterica serovar Enteritidis constitutes a serious global health problem (Pan et al., 2018). This phenomenon is believed to be due to the unregulated use and abuse of antimicrobials especially veterinary drugs including the World Health Organization's critically important antimicrobials for prophylaxis, during the management of diseases or as growth promoters (Azmi et al., 2018; Sharma et al., 2018; Yu et al., 2021). S. Enteritidis is one of the most important serovars shared between humans and animals in most parts of the world (Mohan et al., 2019). It is notorious for its ability to colonize livestock; wild animals as well as the reproductive system of chickens where they persist without any apparent clinical manifestation, thus continuously contaminate eggs and the immediate environment (Campioni et al., 2018; Abdulhaleem et al., 2019). The sustained and undetected presence of these bacteria in food-producing animals makes it possible to cause prolonged epidemics globally, especially where the consumption of poorly cooked poultry egg and meat is common (Salihu et al., 2015; Judd et al., 2019).

Salmonella Enteritidis is one of the major cause of invasive salmonellosis globally, particularly among malnourished children and adults with debilitating illnesses (Stanaway et al., 2019). Population-based estimates of the burden of enteric Salmonella infection in Southeast Asia range from 2.2 to 10.7 cases/100,000 people annually (Whistler et al., 2018). Similarly, S. Enteritidis is reported to account for $38 \%$ of all clinical cases in the Southeast Asia region (Eng et al., 2015). Recent investigations in Thailand on the causes of bacteremia discovered that $S$. Enteritidis accounts for $51.6 \%$ of all cases of invasive non-typhoidal Salmonella infections (Phu Huong Lan et al., 2016; Whistler et al., 2018). In a related investigation to determine the major food pathogens isolated in food materials in China, Salmonella, alongside Vibrio parahaemolyticus, and Campylobacter were among the most prevalent (Paudyal et al., 2018). These observations points to the continued risk humans face from these infectious disease pathogens, particularly where they exhibit antimicrobial resistance (AMR).

Gastro-enteric infection as a result of $S$. Enteritidis has persisted in Malaysia and continues to be a serious public health problem (Thung et al., 2016; Zakaria et al., 2020). The incidence rate has steadily increased in recent years, and this may be attributed to the unhygienic mode of handling and processing of food, especially poultry and poultry products (Thung et al., 2018; Mohan et al., 2019). Although considerable measures to improve food safety has been put in place by the relevant public health authorities, pockets of outbreaks involving large populations continue to be recorded (Balkis et al., 2017; Packierisamy et al., 2018). Other possible reasons why $S$. Enteritidis continues to be a health burden in Malaysia include the proliferation of rodent reservoirs in poultry farms, households, and public food restaurants, evolutionary selection, and the emergence of antimicrobial-resistance among other evolutionary traits (Porwollik et al., 2005; Betancor et al., 2009; Campioni et al., 2018; Azmi et al., 2021). In Malaysia like many parts of the world, it is believed that resistant $S$. Enteritidis had been introduced into the country through the importation of poultry meats and poultry products from other endemic countries (Elemile et al., 2019). In light of these explanations, and the need to understand the phenotypic and genomic diversity of circulating $S$. Enteritidis strains in Malaysia, a Whole-genome sequencing approach was used to assess the phylogenetic relationships, virulence factor determinants, and the antimicrobial-resistance profile of a collection of $S$. Enteritidis isolated from the Central region of Peninsular Malaysia from 2016 to 2018 , with a view to understand the genetic relationship of this important pathogen across different sources (human clinical samples, live birds, and chicken meat).

\section{MATERIALS AND METHODS}

\section{Salmonella Isolates Selection}

The 82 Salmonella isolates used for this study were obtained from government diagnostic laboratories operating within Peninsular Malaysia as part of an ongoing National Antimicrobial Surveillance Program. The isolates were collected from multiple sources, including humans, poultry, and food. Salmonella was isolated at various time points from 2016 to 2018. The human Salmonella isolates $(n=35)$ were obtained from blood and stool samples collected from clinical cases and provided by the Bacteriology Unit, Institute for Medical Research, Ministry of Health Malaysia. Poultry samples (29) originated from fecal samples from commercial poultry farms provided by the Antimicrobial Surveillance Section, Department of Veterinary Services, Ministry of Agriculture Malaysia. At the same time, the food isolates were obtained from ready to eat food from restaurants and chicken meats from wet markets (18) and were provided by the Food Safety and Quality Division, Ministry of Health Malaysia. All the samples were stored in nutrient agar slants until required for further characterization.

\section{Serotype Prediction and Evaluation of Phenotypic AMR}

Traditional serotype determination, according to the Kauffmann White Scheme and the in silico serotype prediction based on the raw reads and genome assemblies, were conducted using SeqSero2 (SeqSero2 v1.1.0) pipeline (Zhang et al., 2019). While the Kauffmann White Scheme determines Salmonella serovars using standard agglutination (SSI Diagnostica Salmonella Seroquick ID kit, UC Bioscience Sdn, Bhd) method according to their antigenic formula ( $\mathrm{O}$ and $\mathrm{H}$ antigens), the SeqSero 2 software uses a k-mer based algorithm to predict serovars from raw reads which ensures improved serotype prediction from draft genome assemblies (Diep et al., 2019; Zhang et al., 2019).

Similarly, all the Salmonella isolates serotyped using the traditional Kauffmann White Scheme and then subjected to phenotypic antimicrobial susceptibility test using the 
Kirby Bauer Disc Diffusion method. A panel of nine (9) antimicrobials purchased from Oxoid (Thermo Scientific Microbiology Sdn Bhd)including ampicillin (Amp-R $<13 \mathrm{~mm}$ ), chloramphenicol (C-R $<12 \mathrm{~mm})$, gentamicin $(\mathrm{CN}-\mathrm{R}<12 \mathrm{~mm}$ ), streptomycin ( $\mathrm{S}-\mathrm{R}<11 \mathrm{~mm}$ ), sulfamethazine/trimethoprim (SXT-R $<10 \mathrm{~mm}$ ), tetracycline (TE-R $<11 \mathrm{~mm}$ ), ceftiofur (EFT$\mathrm{R}<10 \mathrm{~mm}$ ), cefotaxime (Ctx-R $<22 \mathrm{~mm}$ ), and ciprofloxacin (CIP-R $<15 \mathrm{~mm}$ ) was used (Wayne, 2019). Escherichia coli ATCC25922 was used as internal quality control. The Salmonella isolates with the zone of inhibition within the intermediate range were considered to be susceptible to avoid overestimation of resistance.

\section{Genome Library Preparation, Sequence Assembly, and Annotation}

About $2 \mathrm{ml}$ of an overnight culture (at $37^{\circ} \mathrm{C}$ ) of the Salmonella isolates $(n=82)$ at on Luria-Bertani (L.B.) agar was pelleted by centrifugation at $5000 \times g$ for $10 \mathrm{~min}$. The genomic DNA was extracted using QIAamp DNA Mini Kit (Qiagen), and the purity and concentration were determined using the NanoDrop spectrophotometer (Thermo Fisher Scientific). The Nextera ${ }^{\mathrm{TM}}$ DNA Flex Library Prep Kit was employed for the preparation of the genomic libraries. The whole-genome sequencing was performed on the NextSeq 550 System (Illumina, United States). Sequencing reads obtained from the Illumina NextSeq sequencer were scanned for adapter sequence and low-quality sequence using BBDuk (BBTools version 36), where adapter trimming, quality trimming, contaminant filtering, and read length filtering was done, and part of the reads containing poor quality sequence were removed. The good quality sequencing reads were then assembled using SPAdes (SPAdes version 3.9.0) to obtain contigs (Bankevich et al., 2012).

Due to the different sequence profiles, the gene annotation was achieved by predicting the rRNA genes using RNAmmer (Lagesen et al., 2007), while the tRNA genes were predicted using ARAGORN (Laslett and Canback, 2004). The protein-coding genes were first predicted using Prodigal (Hyatt et al., 2010), and the predicted sequences were used to predict their function by using BLAST (Camacho et al., 2009) and HMMER (Eddy, 1998) to search against various sequence or domain databases.

\section{Comparative Analysis}

All the assembled contig sequences from the 82 Salmonella isolates as well as representative reference genomes of closely related Salmonella species (CP019177.1, CP036165.1, CP036166.1, CP022500.1, CP037917.1, CP019183.1, CP022489.1, CP003278.1, AE006468.2, NC_003197.2, and CP014996.1) obtained from the NCBI database were used to infer the relationship, while Salmonella bongori (NC_015761.1) and E. coli (NC_000913.3) were used as outgroup controls. All the sequences were subjected to comparative studies using the EPInod pipeline developed by BioEasy Sdn Bhd. The software package is capable of evaluating the sequences for average nucleotide identity (ANI), multi-locus sequence typing (MLST), Single Nucleotide Polymorphism (SNP)-based phylogenomics estimation, pan-genome gene conservation analysis, virulence factor (V.F.) detection, and AMR gene detection.
Whole-genome was utilized to deduce the genetic relationship among isolates from different species. The identification of pangenome SNPs and phylogenetic analysis was made using the kSNP3.0 program, as described by Gardner et al. (2015). The FASTA input file of the target genomes for SNPs discovery was first input, and $k$, which represents the length of the flanking sequence, including the SNPs, was specified. Finally, SNPs positions in the finished genomes were found by matching with MUMmer. The phylogenetic tree was inferred using all genome SNPs aligned multiple FASTA files with MEGA7 (Kumar et al., 2016). The maximum-likelihood phylogenetic tree of all SNPs loci was generated using the Jukes-Cantor/GTI model with calculated $\mathrm{SH}$-like branch support (1,000 iterations), not bootstrapping. This is because $\mathrm{SH}$-like branch support is fast and efficient, and is commonly used as the default method of choice for phylogenetic software.

Similarly, the genome Virulence Factor analysis to determine the major virulence factors of the characterized bacterial pathogen was achieved using the virulence factor database (VFDB), which is an integrated and comprehensive online resource for curating information about virulence factors of bacterial pathogens (Chen et al., 2016). The NCBI BLAST tool was used to scan predicted CDS (nucleotides) against VFDB for virulence factors, which represent the measure of the pathogenicity of the isolates. The virulence factors analysis was done in two sets, where set A includes genes with experimentally verified V.F. only while set B covers all genes related to known and predicted V.Fs in the V.F database. Finally, the Genome Antibiotic Resistance to identify the AMR profile of the isolates was streamlined via the abricate program against ResFinder and the Comprehensive Antibiotic Resistance Database (CARD) to screen contigs for AMR.

\section{Correlation Between the Phenotypic and Genotypic AMR Profiles}

The antimicrobial susceptibility pattern of the isolates was determined with the Kirby Bauer disk diffusion test, as mentioned earlier. The AMR pattern of the isolates was correlated with its known corresponding resistance gene for each of the strains detected by the WGS analysis, and the percentage correlation was calculated. This was done by counting the genotypic results and dividing the total by the number of isolates that exhibited phenotypic resistance to determine the sensitivity. While the specificity was calculated by dividing the number of isolates that showed genotypic susceptibility by the total number of isolates showing phenotypic susceptibility (Trevethan, 2017). The percentages of positive predictive values (PPVs) and negative predictive values (NPVs) were also calculated as described by Trevethan (2017).

\section{Statistical Analysis}

The association between AMR determinants and virulence genes is calculated using the Chi-square test, and the $P$-value level $<0.05$ is considered significant. Similarly, the correlation between the phenotypic and genetic AMR was also deduced. A one-way analysis of variance (ANOVA) was also conducted to determine if there is significant difference between the means of the three 
hosts (Human, Poultry and Food), followed by a post hoc for multiple comparisons.

\section{RESULTS}

The Kauffmann White Scheme serotyping tests indicated that all the isolates from the three different sources (human, poultry, and food) belonged to the $S$. Enteritidis serogroup. However, the overall results suggested that SeqSero2 (SeqSero2 v1.1.0) improved the serotype prediction compared to the Kauffmann White Scheme serotyping scheme. Although 93\% of the serotype prediction according to the Kauffmann White Scheme was concordant with the in silico WGS prediction, six isolates were found to conflict as they were predicted to be Brancaster (S6-human), Mbandaka (S18-human), Ohio (S72-poultry), Weltevreden (S77-poultry), and Kentucky (S81-poultry), while S63 (poultry) was found to be either Albany or Duesseldorf because they share the same antigenic formula (8: z4, z24). Additionally, the SeqSero2 also identified one unique isolate with antigenic formula I 4:b:- (S87-poultry), which is not listed in the Kauffmann White Scheme. The antimicrobial susceptibility assay, according to the Clinical and Laboratory Standards Institute (CLSI) standards (Patel et al., 2016), showed that thirty (30) isolates were resistant to at least one of the tested antimicrobial drugs, while twenty-four (24) were resistant to multiple drugs tested (MDR: resistance to three or more antimicrobial classes), and the remaining isolates were all susceptible to the tested antimicrobials. However, only one isolate showed resistance to each of ceftiofur (1 human isolate), cefotaxime (1 human isolate), sulfamethazine/trimethoprim (1 food isolate), gentamicin (1 poultry isolate), and chloramphenicol (1 human isolate), while none of the isolates were resistant to ciprofloxacin. The phenotypic and genotypic antimicrobial susceptibility results are summarized in Tables 1, 2.

The pattern of resistance showed that tetracycline had the highest frequency $(37 / 82,45.12 \%)$, and isolates from human samples showed the highest rate with $17 / 35$ followed by poultry $11 / 29$ and then food $9 / 18$ (Figure 1). The second drug with the highest resistance rate is ampicillin with $4 / 35$ (11.43\%) for human, 5/29 (17.24\%) for poultry, and 0/18 (0.00\%) for food isolates respectively (Figure 2). However, the chi-square statistic to check for any association between the resistances observed and the source of the samples (human, poultry, or food) was found to be 1.772 , and the $p$-value was 0.412 . This signifies that the association is not significant at $p<0.05$. Similarly, no statistically significant difference was observed based on the ANOVA with a $p$-value of $0.704(p>0.05)$.

A total of 19 antimicrobial resistance (A.R) genes corresponding to the nine drugs used in the disc diffusion assay were evaluated from the whole genome sequence data. The aminoglycoside resistance gene $a a c\left(6^{\prime}\right)$-ly was detected in 79 of the 82 isolates $(96.34 \%)$. This observation is in contrast to the phenotypic analysis, which showed that only one gentamicin-resistant isolate (1.22\%) was found among all the

TABLE 1 | Distribution of phenotypic antimicrobial resistance (AMR) profile among S. Enteritidis isolates.

\begin{tabular}{|c|c|c|c|c|c|c|c|c|c|}
\hline Isolates & \multicolumn{9}{|c|}{ Antimicrobial Resistance (\%) } \\
\hline Food & 0/18 (0.00\%) & $0 / 18(0.00 \%)$ & 0/18 (0.00\%) & 0/18 (0.00\%) & $1 / 18(5.56 \%)$ & 9/18 (50.00\%) & 0/18 (0.00\%) & 0/18 (0.00\%) & $0 / 18(0.00 \%)$ \\
\hline Human & 4/35 (11.43\%) & $1 / 35(2.86 \%)$ & 0/35 (0.00\%) & $1 / 35(2.86 \%)$ & 0/35 (0.00\%) & $17 / 35$ (48.57\%) & $1 / 35(2.86 \%)$ & $1 / 35(2.86 \%)$ & 0/35 (0.00\%) \\
\hline Overall & 9 (10.98\%) & $1(1.22 \%)$ & $1(1.22 \%)$ & 2 (2.44\%) & $1(1.22 \%)$ & $37(45.12 \%)$ & $1(1.22 \%)$ & $1(1.22 \%)$ & 0 (0.00\%) \\
\hline
\end{tabular}

The antimicrobial susceptibility/resistance was determined based zone of inhibition according to the CLSI standard. Ampicillin (AMP), chloramphenicol (C), gentamicin (C.N.), streptomycin (S), sulfamethazine/trimethoprim (SXT), tetracycline (T.E.), ceftiofur (EFT), cefotaxime (CTX), and ciprofloxacin (CIP).

TABLE 2 | Distribution of genotypic AMR genes among S. Enteritidis isolates.

\begin{tabular}{|c|c|c|c|c|c|c|c|c|c|}
\hline \multirow{2}{*}{$\begin{array}{l}\text { Source of } \\
\text { isolate }\end{array}$} & \multicolumn{9}{|c|}{ Antimicrobial Resistance (\%) } \\
\hline & $\begin{array}{l}\text { AMP (TEM } \\
\text { 33; TEM 4) }\end{array}$ & C (floR) & $\begin{array}{l}\mathrm{CN}\left(\operatorname{aac}\left(6^{\prime}\right)-l y\right. \\
\operatorname{aad} A)\end{array}$ & $\begin{array}{l}\text { S (strA; } \\
\text { strB) }\end{array}$ & $\begin{array}{l}\text { SXT (sul1; } \\
\text { sul2; dfrA14; } \\
\text { dfrA15) }\end{array}$ & $\mathrm{TE}($ tetA; tetC) & $\begin{array}{l}\text { EFT } \\
\text { (blaCMY-2; } \\
\text { blaTEM-1) }\end{array}$ & $\begin{array}{l}\text { CTX } \\
\text { (bla-TEM, } \\
\text { bla-CTX-M) }\end{array}$ & $\begin{array}{l}\text { CIP } \\
\text { (qnrS1; } \\
\text { qnrD1) }\end{array}$ \\
\hline Poultry & $\begin{array}{l}6 / 29 \\
(20.69 \%)\end{array}$ & $\begin{array}{l}-/ 29 \\
(0.00 \%)\end{array}$ & 28/29 (96.55\%) & $\begin{array}{l}1 / 29 \\
(3.45 \%)\end{array}$ & $-/ 29(0.00 \%)$ & $10 / 29(34.48 \%)$ & $-/ 29(0.00 \%)$ & $-/ 29(0.00 \%)$ & $\begin{array}{l}1 / 29 \\
(3.45 \%)\end{array}$ \\
\hline Human & $\begin{array}{l}3 / 35 \\
(8.57 \%)\end{array}$ & $\begin{array}{l}1 / 35 \\
(2.86 \%)\end{array}$ & $34 / 35$ (97.14\%) & $\begin{array}{l}-/ 35 \\
(0.00 \%)\end{array}$ & 2/35 (5.71\%) & $19 / 35$ (54.29\%) & $-/ 35(0.00 \%)$ & $1 / 35(2.86 \%)$ & $\begin{array}{l}2 / 35 \\
(5.71 \%)\end{array}$ \\
\hline Overall & $9(10.98 \%)$ & $1(1.22 \%)$ & 79 (96.34\%) & $1(1.22 \%)$ & $3(3.66 \%)$ & $38(46.34 \%)$ & $0(0.00 \%)$ & $1(1.22 \%)$ & $3(3.66 \%)$ \\
\hline
\end{tabular}

The table presents multiple genes that confer resistance against the antimicrobial drugs used for the antimicrobial susceptibility test (AST) analysis. Ampicillin (Amp), chloramphenicol (C), gentamicin (C.N.), streptomycin (S), sulfamethazine/trimethoprim (SXT), tetracycline (T.E.), ceftiofur (EFT), cefotaxime (CTX), and ciprofloxacin (CIP). 


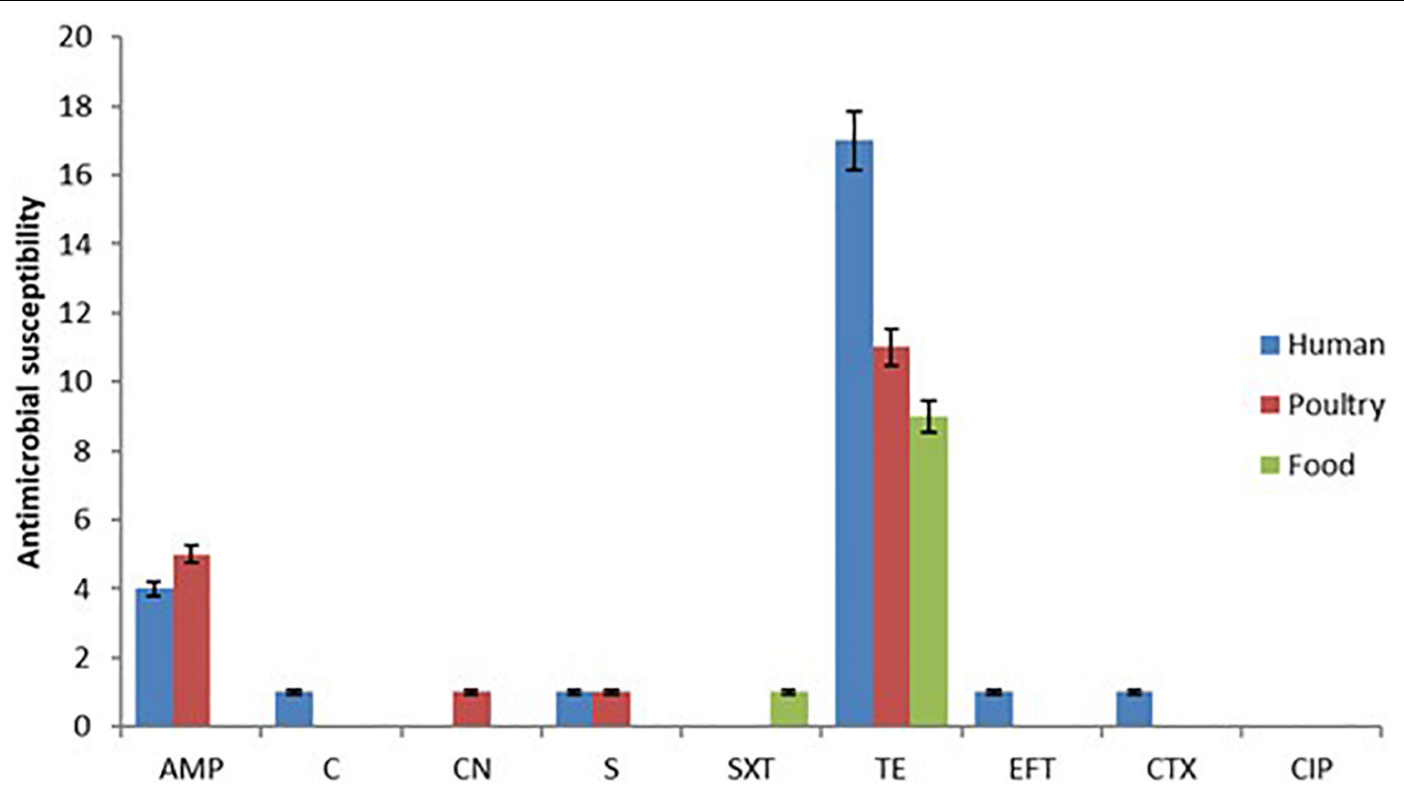

FIGURE 1 | Showing the antimicrobial susceptibility test results based on the Kirby Bauer disk diffusion method indicating high resistance to tetracycline and ampicillin.

isolates. Although, the detection of the tetracycline-resistant genes (tet $A$, tet $C$, and tetD) 38/82 (46.34\%) was found to correspond with the phenotypic result for tetracycline $37 / 82$ (45.12\%), albeit with the genotypic detection being higher by one isolate (Figure 2). However, the predominant tetracycline resistance encoding gene was tet $C$, with only one isolate possessing the tet $A$ gene.

Ten (10) isolates were resistant to beta-lactams (10.98\%), and the two genes encoding beta-lactamases identified in these isolates were TEM33 (10/82), and TEM4 (10/82). Not a single isolate was found to show resistance to the quinolone ciprofloxacin based on the disc diffusion method. However, the genes $q n r S$, and $q n r D 1$ were detected from two human isolates and one poultry isolate. Other resistance genes identified by the whole-genome AMR analysis are $d f r A 14$ (3/82), $d f r A 15$ (3/82), and sul2 (2/82) for trimethoprim and sulfonamide resistance as well as one floR gene (1/82) that codes for chloramphenicol resistance.

\section{Correlation Between AMR Based on Kirby Bauer Disc Diffusion Test and Whole-Genome Sequence AMR Analysis}

The data generated from the whole-genome sequence AMR analysis was correlated with the phenotypic AMR to evaluate the ability of the whole-genome sequence data to predict the phenotypic AMR profiles obtained by the disc diffusion method. The predominant phenotypic AMR resistance observed was resistance against tetracycline (45.12\%) and ampicillin (10.98\%). However, the analysis did not include ciprofloxacin because none of the isolates studied showed phenotypic resistance to ciprofloxacin. Generally, a strong correlation between the phenotypic resistance by disc diffusion and the AMR gene prediction by WGS was observed, except for gentamicin, which was discordant (Table 3).

The overall sensitivity for the WGS based AMR prediction across all the antimicrobial agents evaluated was $50.97 \%$, while the specificity was $55.73 \%$. The post-test probability of the AMR prediction, which measures how well the technique predicts the resistance accurately, was $20.12 \%$, while the NPV was $91.78 \%$.

\section{Analysis of S. Enteritidis Virulence Determinants}

For this article, only those virulence genes that are associated with experimentally verified virulence factors were considered. The virulence factors included bacterial toxins, cell surface proteins that mediate the attachment, cell surface carbohydrates, and proteins that protect the bacterium and hydrolytic enzymes that may contribute to the pathogenicity of the bacteria. These genes were analyzed using the BLAST program against the VFDB. The parameters used were the Percent identity cutoff (50), Percent query coverage per high-scoring segment pair (hsp) (50), and the number of aligned sequences to keep (Chen et al., 2016). A total of 121 virulence genes were detected by the BLAST search against VFDB. The virulence-associated determinants identified in this study include a group of transport, adhesion and some effector proteins such as; Salmonella virulence plasmid determinant protein, $\mathrm{Mg}^{2+}$ transport protein, Intiminlike protein, resistance to compliment killing protein, type III secretion system effector and plasmid-encoded fimbria protein, among other (Supplementary Table 1).

The virulence genes identified in this study are predominantly genes belonging to the type III secretion system (T3SS), which is encoded by the Salmonella pathogenicity sequence 1 (SPI-1) and Salmonella pathogenicity sequence 2 (SPI-2). The type III 


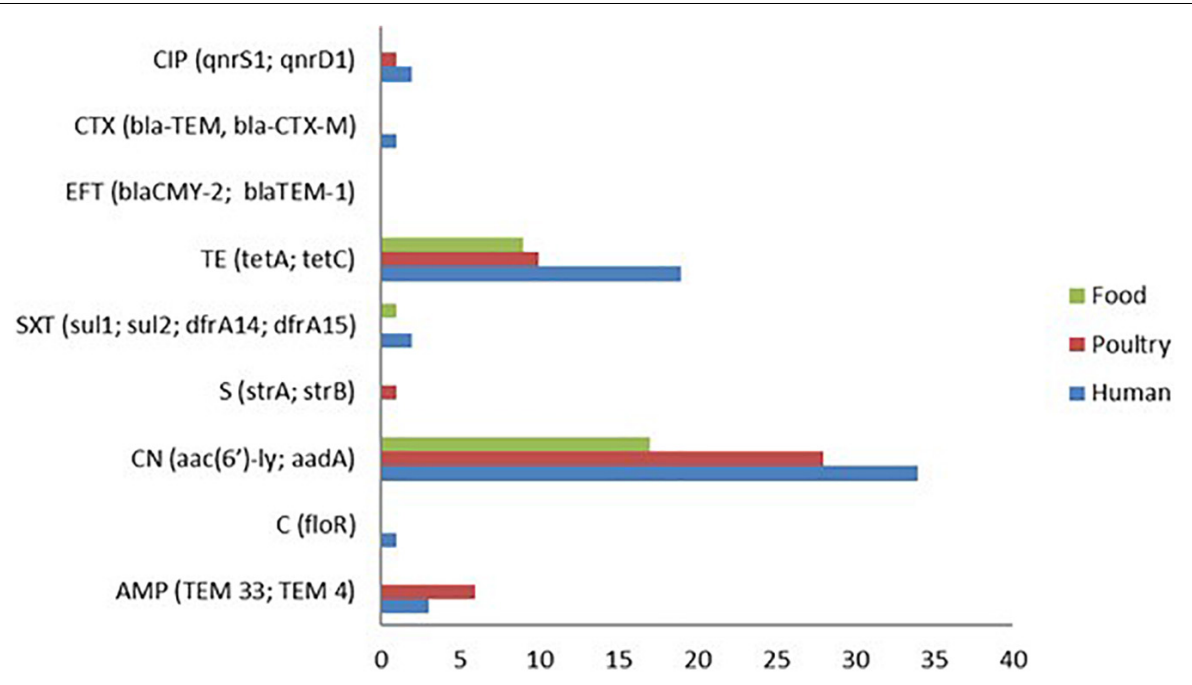

FIGURE 2 | Genotypic antimicrobial resistance (AMR) patterns of $S$. Enteritidis isolates showing aminoglycoside resistance genes (aac ( $\left.6^{\prime}\right)$-ly) and aadA as the predominant resistance genes detected followed by tetracycline (tet $A$ and tet $C$ ) genes.

TABLE 3 | Correlation between AMR phenotype by Kirby Bauer method and genotypic AMR WGS analysis for $S$. Enteritidis isolates ( $n=82)$.

\begin{tabular}{|c|c|c|c|c|c|c|c|c|}
\hline \multirow[t]{2}{*}{ Antimicrobials } & \multicolumn{2}{|c|}{ Resistant isolates } & \multicolumn{2}{|c|}{ Susceptible isolates } & \multirow[t]{2}{*}{ Sensitivity (\%) } & \multirow[t]{2}{*}{ Specificity (\%) } & \multirow[t]{2}{*}{ PPV (\%) } & \multirow[t]{2}{*}{ NPV (\%) } \\
\hline & Genotypic & Phenotypic & Genotypic & Phenotypic & & & & \\
\hline AMP & 9 & 9 & 73 & 73 & 10.98 & 89.02 & 50.00 & 50.00 \\
\hline C & 1 & 1 & 81 & 81 & 1.22 & 98.78 & 50.00 & 50.00 \\
\hline $\mathrm{CN}$ & 79 & 1 & 3 & 81 & 96.34 & 98.78 & 98.75 & 96.43 \\
\hline S & 1 & 2 & 81 & 80 & 1.22 & 97.56 & 33.33 & 49.69 \\
\hline SXT & 3 & 1 & 79 & 81 & 3.66 & 98.78 & 75.00 & 50.63 \\
\hline TE & 38 & 37 & 44 & 43 & 46.34 & 53.75 & 50.67 & 49.43 \\
\hline EFT & 0 & 1 & 82 & 81 & 0.00 & 98.78 & 0.00 & 49.69 \\
\hline CTX & 1 & 1 & 81 & 81 & 1.22 & 98.78 & 50.00 & 50.00 \\
\hline Total & & & & & 20.12 & 91.78 & 50.97 & 55.73 \\
\hline
\end{tabular}

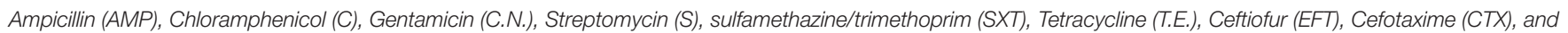
Ciprofloxacin (CIP), PPV-Positive Predictive Value, NPV-Negative Predictive Value.

SPI 1 genes include; system effector (SteA and SteB), type III secretion system accessory cytosolic protein (OrgA and OrgC), type III secretion system regulatory protein (InvA-InvJ), type III secretion system export apparatus switch protein (SpaO-SpaS), and type III secretion system hydrophilic translocator, and pore protein (SipA-SipD) (Supplementary Table 1). These genes were detected in all the isolates from the human, poultry, and food samples. Similarly, SPI-2 system effector (SopB, SopD2, and SlrP) chaperone protein-coding (sseB-sseC) SteC, SseK1, SifB, $\mathrm{SseK}$, and type III secretion system gatekeeper ( $\mathrm{SsaH}$-SsaL) were among the genes detected in all the isolates except S63 isolate from poultry swab.

In addition to the genes within the pathogenicity sequences, virulence plasmid (plasmid-encoded fimbriae chaperone protein PefD), ion acquisition (ferrienterobactin outer membrane transporter), fimbriae (long polar fimbrial chaperone protein, type I fimbriae adaptor protein FimF), as well as the flagella and flagellin genes (flagellar motor protein MotA) were all identified. Within the fimbrial adherence determinants, are genes that codes for the curli fimbriae and curli assembly protein CsgC that mediate the binding to various serum and tissues matrix proteins (Thomas et al., 2017). Other important virulence determinants detected from most of the isolates after the BLAST analysis within the VFDB software are; the antimicrobial peptide resistance protein Mig-14, $\mathrm{Mg}^{2+}$ transport protein $\mathrm{MgtBC}$, and the Salmonella plasmid virulence which is responsible for the regulation of spv operon.

\section{Phylogenomics of S. Enteritidis}

Phylogenetic analysis for the 82 genome sequence (WGS data attached as a Supplementary Document 2 - https://submit.ncbi. nlm.nih.gov/wgs_common/report/SUB8477823) was conducted using the maximum likelihood method. The analysis included the sequence from the 35 human isolates, 18 isolates from food, and 31 isolates obtained from poultry cloacal swabs and an $S$. Brancaster, S. Ohio, S. Mbandaka, S. Kentucky, S. Weltevreden, S. Typhimurium, S. Bongori, and E. coli K12 control genome. Two bifurcating clades and one monophyletic clade from the 
ancestral node were confirmed from the phylogenetic tree. The rooted $S$. Enteritidis tree indicated multiple bifurcating patterns with varying levels of diversity and placed both $S$. Enteritidis isolates from humans, poultry, and food samples into distinct monophyletic clades, suggesting that the isolates evolved into distinct evolutionary lineages from their common phylogenetic ancestor. The ancestral node branched into two with the E. coli (clade A) outgroup diverging away from the Salmonella group (clade B). Subsequently, clade B bifurcated into sub-clade B1 occupied by S. Bongori and B2 (S. enterica), which later splits into multiple clusters with variable genetic properties compared to the reference genomes.

The phylogenetic analysis revealed that sub-clade B2-I contained S6 and S18 from human clinical samples, which clustered with $S$. Brancaster and $S$. Mbandaka, respectively. While S63, S72, S77, and S81 clustered with S. Albany, S. Ohio, S. Weltevreden, and $S$. Kentucky, respectively (sub-clade B2-I). The clustering of these isolates with the reference genome conforms with the SeqSero serotype allocation. Important to note that while the SeqSero analysis identified that S63 shared the same antigenic formula with $S$. Albany and $S$. Duesseldorf, the phylogenetic tree showed it is more genetically related with $S$. Albany rather than S. Duesseldorf. S. Typhi, which is typhoidal Salmonella, was found to share some genetic relationship with $S$. Ohio, $S$. Enteritidis, and $S$. Typhimurium having branched from the same bifurcation. Sub-clade B2-II, on the other which contained the largest convergence of the isolates, implies close genetic relation across the different sources.

\section{DISCUSSION}

Salmonella serotyping using the traditional phenotypic method was the first analysis conducted in this study because of its importance in the characterization of Salmonella, especially during epidemiological investigations and surveillance programs (Diep et al., 2019). However, because of the ease and superior predictability of WGS based in silico methods, SeqSero 2 was used to confirm the phenotypic serotyping (Zhang et al., 2019). The results show that the SeqSero2 correctly identified $S$. Enteritidis in concordance with the Kauffmann White Scheme result with 93\% similarity. However, the SeqSero2 was able to identify six serovars other than $S$. Enteritidis (Brancaster, Mbandaka, Ohio, Weltevreden, and Kentucky), including Albany and Dusseldorf which had the same antigenic formula (I 4:b:-), while one was not available in the database. This finding agrees with earlier studies that indicated that the same antigenic formula could be shared by different subspecies (Diep et al., 2019). In silico WGSbased serotyping has been shown to perform better in identifying Salmonella serotype diversity compared to the traditional method and has become popular among molecular epidemiologists and public health scientists (Diep et al., 2019; Elnekave et al., 2020).

The present investigation also evaluated AMR determinants, virulence factor genes, and phylogenetic relationships of the isolates using a whole-genome sequencing approach. This is because comparative genomics studies have indicated that host specificity (humans, animals, birds, and environment) is a very important factor driving the evolution of new lineages of enteric Salmonella species (Tasmin et al., 2017; Deblais et al., 2018). Secondly, the choice of WGS is because its application permits a broader inference of pathogen characterization, including the prediction of antibiotic resistance and virulence determinants from the sequence (Gupta et al., 2019). The 82 S. Enteritidis, S. Brancaster, S. Mbandaka, S. Albany, S. Ohio, S. Weltevreden, and $S$. Kentucky isolates identified in this study were cultured from multiple sources, including human clinical samples (blood and feces), poultry (cloacal swab), and food (chicken meat). Upon obtaining the whole genome sequence, EPInod pipeline was used for the analysis of AMR profile, virulence determinants, and the SNP-based phylogenetic analysis.

Antimicrobial usage represents a very critical factor in the emerging public health crisis due to antibiotic resistance. Its extensive application in food animal production to manage clinical infections, as prophylaxis to prevent and control livestock diseases, as well as its use as an additive in feed to enhance growth and productivity have all played roles in the emergence of resistance (Salihu et al., 2013; Wang et al., 2019; Van et al., 2020; Ma et al., 2021). In this study, tetracycline (45.12\%) and ampicillin (11.43\%) were identified as drugs with the highest phenotypic resistance rate. Similarly, resistance to these drugs was highest among the human isolates (17/35), followed by food isolates (9/18) and then poultry (11/29). These antimicrobials are among those that have been declared critical by the WHO and their use in animals has been restricted. This finding implies that some of these legislation is not being observed and this could lead to the loss of potency of the drugs and progressive emergenc of antimicrobial resistant pathogens. Also, $29.27 \%$ of the isolates comprising of $S$. Enteritidis, $S$. Mbandaka, S. Ohio, S. Kentucky, and S. Brancaster exhibited multiple resistances to 2 or more drugs. According to studies sanctioned by the Department of Veterinary Services Malaysia under the Livestock Farm Practices Scheme (SALT) program, for Salmonella species isolated from chicken cloacal swabs revealed that $13.5 \%$ of the Salmonella isolates were resistant to tetracycline, $5.4 \%$ to polymyxin and erythromycin. This is similar to the investigation conducted in China where tetracycline resistance was found to predominate among Salmonella isolates obtained from slaughtered pigs (Wu et al., 2021). In comparison, 2.7\% were resistant to chloramphenicol and trimethoprim (Azmi et al., 2018). Despite the resistance observed in this study, the association between the resistance rate and the source of the sample as well as statistical significance based on ANOVA between their means was found not to be statistically significant $(p>0.05)$. Other drugs that were found to exhibit resistance were chloramphenicol among human isolates $(2.86 \%)$, gentamicin in poultry $(3.45 \%)$, sulfamethazine/trimethoprim in food (5.56\%), ceftiofur and cefotaxime both in human $(2.86 \%)$. In a related study conducted in the East Coast of Peninsula Malaysia, Salmonella isolates from broiler chicken were found to exhibit resistance to chloramphenicol (76.2\%), sulfamethoxazole/trimethoprim (42.9\%) (Ibrahim et al., 2021). This further highlights the crucial role of poultry and poultry products in the dissemination of resistant Salmonella pathogens to humans and other susceptible animal species. 
On the other hand, the WGS analysis of AMR genes correlated significantly by identifying some genes that encode resistance to the majority of the phenotypic resistance observed. These included tetracycline, ampicillin, streptomycin, sulfamethazine/trimethoprim, cefotaxime, and ciprofloxacin resistance genes such as tet $A$, tetC, TEM33, TEM4, floR, aac(6')$l y$, strA, sul1, dfrA 14, dfrA15, blaCTX-M, and qnrS1, respectively (Table 2). Although no phenotypic resistance was observed for gentamicin, the AMR genotype result showed that the gentamicin gene $\left(a a c\left(6^{\prime}\right)-l y\right)$ was the most frequent (96.34\%) with isolates from a human source having the highest percentage (97.14\%). This observation goes to show WGS as an excellent tool for the accurate prediction of antimicrobial-resistant phenotype in human, animal, and environment samples (Pornsukarom et al.,
2018; Liu et al., 2021). The gene $\operatorname{aac}\left(6^{\prime}\right)$-ly is one of the important chromosomal genes responsible for the enzymatic modification of aminoglycoside leading to the development of resistance (Odumosu et al., 2015; El-Badawy et al., 2017). However, it is important to note that mechanism for aminoglycoside resistance include among others decreased uptake and accumulation of the drug in the bacterial pathogen, as well as the expression of aminoglycoside modifying enzymes (AMEs) that causes inactivation of the drug (Mir et al., 2016). Even though, a strong correlation between the phenotypic resistance by disc diffusion and the AMR gene prediction by WGS was observed (sensitivity $20.12 \%$ and specificity $91.78 \%$ ), the result for gentamicin was discordant (Table 3). The most common genes conferring resistance to $\beta$-lactam antibiotics in this study were TEM 33

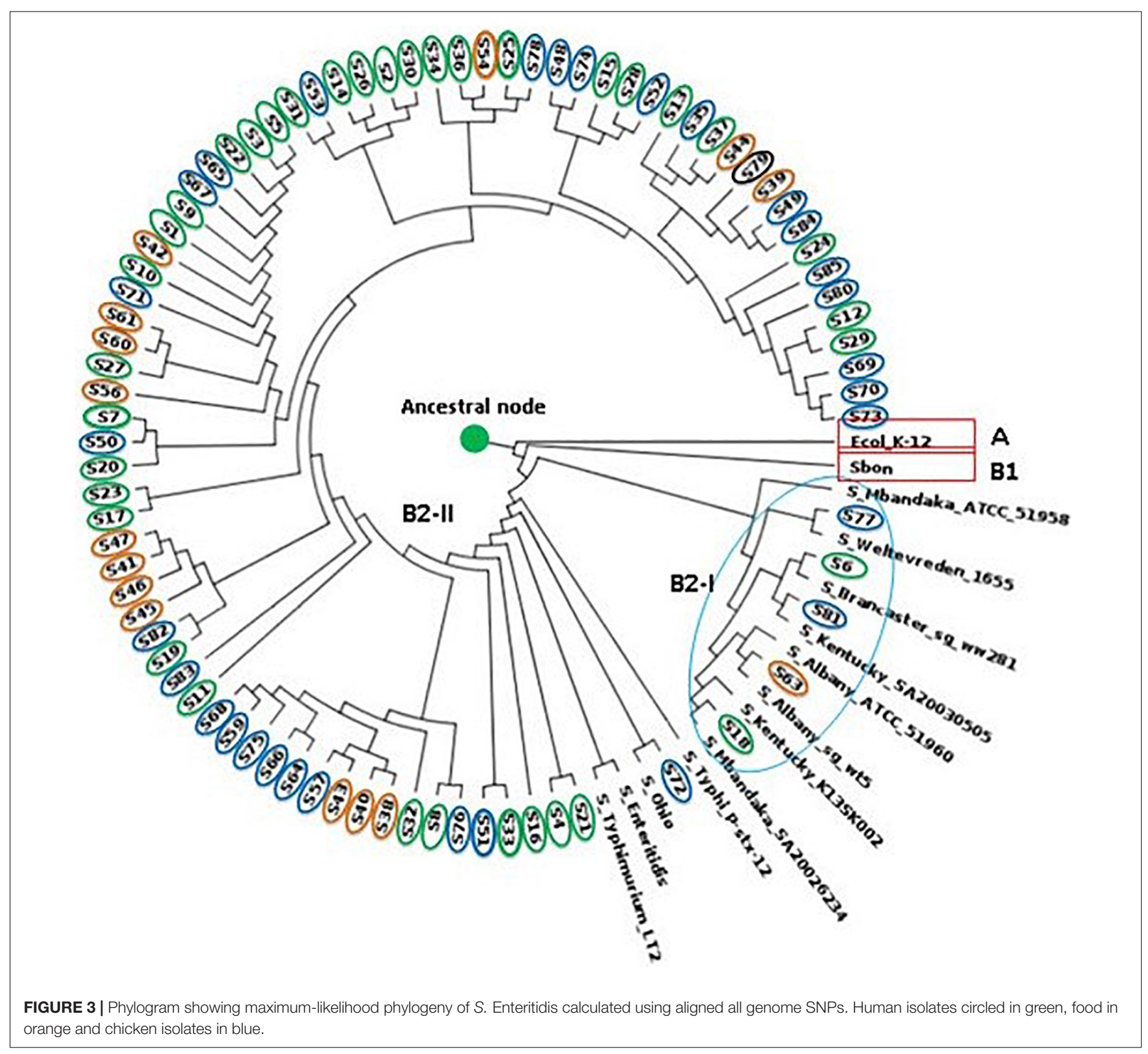


and TEM 4 without corresponding phenotypic consequence. Qualitative determination of antimicrobial susceptibility based on the zone of inhibition is prone to error due to the instability of antibiotic-containing discs under varying temperature and humidity, interference of the functionality of some antimicrobials by the components of growth agar, and the possibility that an isolate may be falsely classified as susceptible (Neuert et al., 2018; Srivastava et al., 2020). This may explain the relative number of mismatches observed in the present study. Moreover, many of the resistance genes detected by the WGS algorithm are plasmid-encoded. In other words, bacterial plasmids are known to commonly cure during storage and sub-culture of the isolates, such that genes detected during sequencing might have become altered (Neuert et al., 2018). Furthermore, Salmonella has been shown to possess silent resistance genes, as well as variants $\left(\operatorname{aac}\left(6^{\prime}\right)\right)$, which only become transcriptionally active in rare cases (Heider et al., 2009; Frye and Jackson, 2013; Adesiji et al., 2014).

The WGS analysis revealed multiple virulence genes among the Salmonella isolates across the different sources (Supplementary Table 2). These genes included the type III secretion system (T3SS) located within the Salmonella Pathogenicity Sequence I and II, intimin-like proteins, plasmidencoded fimbriae chaperone protein, host recognition/invasion determinants, as well as magnesium uptake, iron acquisition factors. These genes are responsible for Salmonella infectivity, transmission, and survival (Pornsukarom et al., 2018). The result of the present study indicates that the $S$. Enteritidis isolated from the poultry cloacal swabs and food sources possessed the same virulence genes as the human clinical isolates. Virulence factors such as adhesins, toxins, and iron ion transport systems are responsible for the adhesion of pathogens to epithelial cells of intestines, which constitute an essential colonization factor during intestinal infections (Sarowska et al., 2019). The detection of a high number of plasmid encoding virulence genes in food, and especially poultry, may serve as a means of transmission of these resistance factors to humans, as observed in this study. The detection of fimbrial genes and a host of other plasmid-encoded genes within the pathogenicity sequences 1-2 among the isolates in this study may signify the potentials of these isolates to cause severe humans infection. Hence, this report will provide valuable reference data that will assist in future investigations of human Salmonella infection. In the same vein, the phylogenetic tree obtained from SNPs exhibited diverse clustering of the isolates with an initial one singleton and two clades that subsequently bifurcated. Phylogenetic analysis based on WGS-derived has proven to be a superior cluster resolution method compared to many of the standard subtyping methods (Mohammed and Thapa, 2020; Ford et al., 2021). In order to determine the genetic relatedness among the isolates and infer their evolutionary history based on a genome-wide scale, across the genomes of all $S$. Enteritidis and closely related serovars were identified. Phylogenetic analysis based on the maximum likelihood tree produced strong support for the monophyly of the Salmonella isolates with $S$. Enteritidis being the predominant (Figure 3). Within the $S$. Enteritidis isolates from the different sources, a split that delineates two sister lineages, Clade B1 and B2 was observed. At the same time, Clade A consisted of 1 branch occupied by the E. coli K12 outgroup, Clade B comprised of the $S$. bongori reference strain and the other members of the S. enterica serogroup. Sub-clade B2-I which is a branched form the B1 clade, clustered with five of the isolates (S77, S6, S81, S63, and S18) which shared same branches with $S$. Mbandaka, S. Weltevreden, S. Brancaster, S. Kentucky, and S. Ohio implying close genetic relationship as determined via the SeqSero serotyping. The major bifurcating clade B2-II, on the other hand, contained one monophyletic clade occupied by $S$. Bandaka and another highly branched sub-clades containing isolates from all the three sources. The fact that samples from poultry, human and food as well as the control S. Typhimurium, and $S$. Typhi were located in this well-supported sub-clade may indicate similarity in their gene sequence that can be attributed to convergent evolution due to adaptation to the various sources as earlier reported by Didelot et al. (2007). As deduced from the phylogenetic tree in Figure 3, though the split of clade B is suggestive of allele sharing, possibly via horizontal gene transfer and homologous recombination between the clades, which is a common phenomenon in enteric bacteria (Lawrence and Retchless, 2009; Hall et al., 2020). However, the gene transfer may not have occurred to the extent that divergence between the two clades is eliminated. This is in concordance to divergent lineages observed in $E$. coli following high recombination (Touchon et al., 2009).

\section{CONCLUSION}

An insight into the genetic profile of enteric $S$. Enteritidis to assess the evolutionary and genetic diversity based on SNPs phylogenomics, as well as understand the virulence and resistance pattern using WGS, was undertaken. The aim was to understand further the genomic diversity existing among $S$. Enteritidis from different host species. The potential transmissibility of genetic elements was evident due to the clustering of multiple isolates from all the three sources. Most of the virulence factors were also detected from the genome of all the isolates across the human, poultry, and food isolates. However, while the genotypic AMR was mostly congruent with the phenotypic profiles of the isolates, slight mismatches were observed with particular reference to gentamicin. This discrepancy was attributed to their silent nature of the aac $\left(6^{\prime}\right)$-ly gene whose transcription occurs intermittently. As a whole, the WGS analysis produced a comprehensive understanding of the resistance profile of S. Enteritidis and the ability of the bacteria to adapt to different host species. However, a more in-depth study, including other Salmonella serovars, will be of immense benefit to the understanding of the adaptability and survival mechanism in Salmonella serovars.

\section{LIMITATION OF THE STUDY}

This investigation was not able to ascertain the geographical details of the various samples/sources because the isolates were 
donated as part of stock cultures covering the study period by the agencies involved with the Malaysian National Antimicrobial Resistance Surveillance Program.

\section{DATA AVAILABILITY STATEMENT}

The datasets presented in this study can be found in online repositories. The names of the repository/repositories and accession number(s) can be found in the article/ Supplementary Material.

\section{AUTHOR CONTRIBUTIONS}

ZZ, LH, SAH, and NA: conceptualization. ZS, NMS, and RA: methodology. ZZ, NMS, and BG: software sequence analysis and interpretation. $\mathrm{ZZ}$ and $\mathrm{BG}$ : writing-original draft preparation. ZZ, BG, LH, NA, SAH, ZS, NMS, and RA: writing-review and editing. ZZ: funding acquisition. ZZ and LH: supervision.

\section{REFERENCES}

Abdulhaleem, N., Garba, B., Younis, H., Mahmuda, A., Hamat, R. A., Majid, R. B. A., et al. (2019). Current trend on the economic and public health significance of salmonellosis in Iraq. Adv. Anim. Vet. Sci. 7, 484-491. doi: 10.17582/journal.aavs/2019/7.6.484.491

Adesiji, Y. O., Deekshit, V. K., and Karunasagar, I. (2014). Antimicrobial-resistant genes associated with Salmonella spp. isolated from human, poultry, and seafood sources. Food Sci. Nutr. 2, 436-442. doi: 10.1002/fsn3.119

Azmi, M., Hassali, A., Rhu, H., Ashutosh, Y., Verma, K., Hussain, R., et al. (2018). Antibiotic Use in Food Animals: Malaysia Overview. Malaysia: School of Pharmaceutical Sciences Universiti Sains Malaysia 11800 Minden.

Azmi, M., Hassali, A., Rhu, H., Ashutosh, Y., Verma, K., Hussain, R., et al. (2021). Antibiotic Use in Food Animals: Malaysia Overview. Available online at: www.reactgroup.org [accessed July 25, 2021]

Balkis A. B. K. A., Liza, L, Anita, S. A. B. D. S., Norafidah, A., Rashid, W. M., Wan, M., et al. (2017). A Large Common Source Outbreak of Salmonella typhimurium Linked to Kuala Terengganu Night Markets, Malaysia, 2014. OSIR J. 10, 1-7.

Bankevich, A., Nurk, S., Antipov, D., Gurevich, A. A., Dvorkin, M., Kulikov, A. S., et al. (2012). SPAdes: A new genome assembly algorithm and its applications to single-cell sequencing. J. Comput. Biol. 19, 455-477. doi: 10.1089/cmb.2012. 0021

Betancor, L., Yim, L., Fookes, M., Martinez, A., Thomson, N. R., Ivens, A., et al. (2009). Genomic and phenotypic variation in epidemic-spanning Salmonella enterica serovar Enteritidis isolates. BMC Microbiol. 9:237. doi: 10.1186/14712180-9-237

Camacho, C., Coulouris, G., Avagyan, V., Ma, N., Papadopoulos, J., Bealer, K., et al. (2009). BLAST+: Architecture and applications. BMC Bioinform. 10:421. doi: 10.1186/1471-2105-10-421

Campioni, F., Cao, G., Kastanis, G., Janies, D. A., Bergamini, A. M. M., Rodrigues, D. D. P., et al. (2018). Changing of the Genomic Pattern of Salmonella Enteritidis Strains Isolated in Brazil over a 48 year-period revealed by Whole Genome SNP Analyses. Sci. Rep. 8, 1-7. doi: 10.1038/s41598-018-28 844-6

Chen, L., Zheng, D., Liu, B., Yang, J., and Jin, Q. (2016). VFDB 2016: Hierarchical and refined dataset for big data analysis - 10 years on. Nucleic Acids Res. 44, D694-D697. doi: 10.1093/NAR/GKV1239

Coburn, B., Grassl, G. A., and Finlay, B. B. (2007). Salmonella, the host and disease: A brief review. Immunol. Cell Biol. 85, 112-118. doi: 10.1038/sj.icb.7100007

Deblais, L., Lorentz, B., Scaria, J., Nagaraja, K. V., Nisar, M., Lauer, D., et al. (2018). Comparative Genomic Studies of Salmonella Heidelberg Isolated

\section{FUNDING}

This research was funded by the UPM-Bioeasy Sdn Bhd Matching Grant 6300920 and UPM Putra Grant 9300438.

\section{ACKNOWLEDGMENTS}

The authors wish to acknowledge the contribution of the Ministry of Health and the Department of Veterinary Services, Malaysia, for the donation of isolates as well as expertise during the course of this research and BioEasy Sdn Bhd for the analysis of the WGS data.

\section{SUPPLEMENTARY MATERIAL}

The Supplementary Material for this article can be found online at: https://www.frontiersin.org/articles/10.3389/fmicb. 2021.652642/full\#supplementary-material

From Chicken- and Turkey-Associated Farm Environmental Samples. Front. Microbiol. 9:1841. doi: 10.3389/FMICB.2018.01841

Didelot, X., Achtman, M., Parkhill, J., Thomson, N. R., and Falush, D. (2007). A bimodal pattern of relatedness between the Salmonella Paratyphi A and Typhi genomes: Convergence or divergence by homologous recombination? Genome Res. 17, 61-68. doi: 10.1101/gr.5512906

Diep, B., Barretto, C., Portmann, A.-C., Fournier, C., Karczmarek, A., Voets, G., et al. (2019). Salmonella Serotyping; Comparison of the Traditional Method to a Microarray-Based Method and an in silico Platform Using Whole Genome Sequencing Data. Front. Microbiol. 10:2554. doi: 10.3389/fmicb.2019.02554

Eddy, S. R. (1998). Profile hidden Markov models. Bioinformatics 14, 755-763. doi: 10.1093/bioinformatics/14.9.755

El-Badawy, M. F., Tawakol, W. M., El-Far, S. W., Maghrabi, I. A., Al-Ghamdi, S. A., Mansy, M. S., et al. (2017). Molecular Identification of AminoglycosideModifying Enzymes and Plasmid-Mediated Quinolone Resistance Genes among Klebsiella pneumoniae Clinical Isolates Recovered from Egyptian Patients. Int. J. Microbiol. 2017:8050432. doi: 10.1155/2017/8050432

Elemile, O. O., Raphael, D. O., Omole, D. O., Oloruntoba, E. O., Ajayi, E. O., and Ohwavborua, N. A. (2019). Assessment of the impact of abattoir effluent on the quality of groundwater in a residential area of Omu-Aran. Nigeria. Environ. Sci. Eur. 31:16. doi: 10.1186/s12302-019-0201-5

Elnekave, E., Hong, S. L., Lim, S., Johnson, T. J., Perez, A., and Alvarez, J. (2020). Comparing serotyping with whole-genome sequencing for subtyping of nontyphoidal Salmonella enterica: a large-scale analysis of 37 serotypes with a public health impact in the USA. Microb. Genomics 6, 1-13. doi: 10.1099/MGEN.0. 000425

Eng, S.-K., Pusparajah, P., Ab Mutalib, N.-S., Ser, H.-L., Chan, K.-G., and Lee, L.H. (2015). Salmonella: A review on pathogenesis, epidemiology and antibiotic resistance. Front. Life Sci. 8, 284-293. doi: 10.1080/21553769.2015.1051243

Ford, L., Glass, K., Williamson, D. A., Sintchenko, V., Robson, J. M. B., Lancsar, E., et al. (2021). Cost of whole genome sequencing for non-typhoidal Salmonella enterica. PLoS One 16:e248561. doi: 10.1371/JOURNAL.PONE.0248561

Frye, J. G., and Jackson, C. R. (2013). Genetic mechanisms of antimicrobial resistance identified in Salmonella enterica, Escherichia coli, and Enteroccocus spp. isolated from U.S. food animals. Front. Microbiol. 4:135. doi: 10.3389/ FMICB.2013.00135

Gardner, S. N., Slezak, T., and Hall, B. G. (2015). kSNP3.0: SNP detection and phylogenetic analysis of genomes without genome alignment or reference genome. Bioinformatics 31, 2877-2878. doi: 10.1093/BIOINFORMATICS/ BTV271

Graham, R. M. A., Hiley, L., Rathnayake, I. U., and Jennison, A. V. (2018). Comparative genomics identifies distinct lineages of S. Enteritidis from 
Queensland, Australia. PLoS One 13:e191042. doi: 10.1371/journal.pone. 0191042

Gupta, S. K., Sharma, P., McMillan, E. A., Jackson, C. R., Hiott, L. M., Woodley, T., et al. (2019). Genomic comparison of diverse Salmonella serovars isolated from swine. PLoS One 14:e0224518. doi: 10.1371/journal.pone.0224518

Hall, R. J., Whelan, F. J., McInerney, J. O., Ou, Y., and Domingo-Sananes, M. R. (2020). Horizontal Gene Transfer as a Source of Conflict and Cooperation in Prokaryotes. Front. Microbiol. 11:1569. doi: 10.3389/FMICB.2020.01569

Heider, L. C., Hoet, A. E., Wittum, T. E., Khaitsa, M. L., Love, B. C., Huston, C. L., et al. (2009). Genetic and phenotypic characterization of the blaCMY gene from escherichia coli and salmonella enterica isolated from food-producing animals, humans, the environment, and retail meat. Foodborne Pathog. Dis. 6, 1235-1240. doi: 10.1089/FPD.2009.0294

Hyatt, D., Chen, G. L., LoCascio, P. F., Land, M. L., Larimer, F. W., and Hauser, L. J. (2010). Prodigal: Prokaryotic gene recognition and translation initiation site identification. BMC Bioinformatics 11:119. doi: 10.1186/1471-2105-11-119

Ibrahim, S., Hoong, L. W., Siong, Y. L., Mustapha, Z., Zalati, C. W. S. C. W., Aklilu, E., et al. (2021). Prevalence of Antimicrobial Resistance (AMR) Salmonella spp. and Escherichia coli Isolated from Broilers in the East Coast of Peninsular Malaysia. Antibiotics 10:579. doi: 10.3390/ANTIBIOTICS10050579

Judd, M. C., Hoekstra, R. M., Mahon, B. E., Fields, P. I., and Wong, K. K. (2019). Epidemiologic patterns of human salmonella serotype diversity in the USA, 1996-2016. Epidemiol. Infect. 147:e187. doi: 10.1017/S0950268819000724

Kumar, S., Stecher, G., and Tamura, K. (2016). MEGA7: Molecular Evolutionary Genetics Analysis Version 7.0 for Bigger Datasets. Mol. Biol. Evol. 33, 18701874. doi: $10.1093 / \mathrm{molbev} / \mathrm{msw} 054$

Lagesen, K., Hallin, P., Rødland, A., Staerfeldt, H.-H., Rognes, T., and Ussery, D. W. (2007). RNAmmer: consistent and rapid annotation of ribosomal RNA genes. Nucleic Acids Res. 35, 3100-3108. doi: 10.1093/nar/gkm160

Laslett, D., and Canback, B. (2004). ARAGORN, a program to detect tRNA genes and tmRNA genes in nucleotide sequences. Nucleic Acids Res. 32, 11-16. doi: 10.1093/nar/gkh152

Lawrence, J. G., and Retchless, A. C. (2009). The Interplay of Homologous Recombination and Horizontal Gene Transfer in Bacterial Speciation. Methods Mol. Biol. 532, 29-53. doi: 10.1007/978-1-60327-853-9_3

Liu, Y., Jiang, J., -Dra, A., Li, X., Peng, X., Xia, L., et al. (eds) (2021). Prevalence and genomic investigation of Salmonella isolates recovered from animal food-chain in Xinjiang. China. Food Res. Int. 142:110198. doi: 10.1016/J.FOODRES.2021. 110198

Ma, F., Xu, S., Tang, Z., Li, Z., and Zhang, L. (2021). Use of antimicrobials in food animals and impact of transmission of antimicrobial resistance on humans. Biosaf. Heal. 3, 32-38. doi: 10.1016/J.BSHEAL.2020.09.004

Mir, A. R., Bashir, Y., Dar, F. A., and Sekhar, M. (2016). Identification of genes coding aminoglycoside modifying enzymes in E. coli of UTI patients in India. Sci. World J. 2016:1875865. doi: 10.1155/2016/1875865

Mohammed, M., and Thapa, S. (2020). Evaluation of WGS-subtyping methods for epidemiological surveillance of foodborne salmonellosis. One Heal. Outlook 21, 1-15. doi: 10.1186/S42522-020-00016-5

Mohan, A., Munusamy, C., Tan, Y. C., Muthuvelu, S., Hashim, R., Chien, S. L., et al. (2019). Invasive Salmonella infections among children in Bintulu, Sarawak, Malaysian Borneo: A 6-year retrospective review. BMC Infect. Dis. 19:330. doi: 10.1186/s12879-019-3963-x

Moussa, A. A., Abdi, A. A., Awale, M. A., and Garba, B. (2021). Occurrence and phenotypic characterization of multidrug-resistant bacterial pathogens isolated from patients in a public hospital in Mogadishu. Somalia. Infect. Drug Resist. 14, 825-832. doi: 10.2147/IDR.S275090

Neuert, S., Nair, S., Day, M. R., Doumith, M., Ashton, P. M., Mellor, K. C., et al. (2018). Prediction of phenotypic antimicrobial resistance profiles from whole genome sequences of non-typhoidal Salmonella enterica. Front. Microbiol. 9:592. doi: 10.3389/fmicb.2018.00592

Odumosu, B. T., Adeniyi, B. A., and Chandra, R. (2015). Occurrence of aminoglycoside-modifying enzymes genes (aac(6')-I and ant(2")-I) in clinical isolates of Pseudomonas aeruginosa from Southwest Nigeria. Afri. Heal. Sci. 15, 1277-1281. doi: 10.4314/ahs.v15i4.29

Packierisamy, P. R., Haron, R., Mustafa, M., Mahir, A., Ayob, A., and Balan, V. (2018). Outbreak caused by food-borne Salmonella enterica serovar Enteriditis in a residential school in Perak state. Malaysia in April 2016. Int. Food Res. J. 25, 2379-2384.
Pan, H., Paudyal, N., Li, X., Fang, W., and Yue, M. (2018). Multiple Food-AnimalBorne Route in Transmission of Antibiotic-Resistant Salmonella Newport to Humans. Front. Microbiol. 9:23. doi: 10.3389/FMICB.2018.00023

Patel, J. B., Eliopoulos, G. M., Jenkins, S. G., James Lewis, I. I. F. S., Brandi Limbago, P., Nicolau, D. P., et al. (2016). Performance Standards for Antimicrobial Susceptibility Testing. Clin. Lab. Stand. Inst. 40:1.

Paudyal, N., Pan, H., Liao, X., Zhang, X., Li, X., Fang, W., et al. (2018). A Meta-Analysis of Major Foodborne Pathogens in Chinese Food Commodities Between 2006 and 2016. Foodborne Pathog Dis. 15, 187-197. doi: 10.1089/FPD. 2017.2417

Phu Huong Lan, N., Le, Thi Phuong T, Nguyen, Huu H, Thuy, L., Mather, A. E., Park, S. E., et al. (2016). Invasive Non-typhoidal Salmonella Infections in Asia: Clinical Observations, Disease Outcome and Dominant Serovars from an Infectious Disease Hospital in Vietnam. PLoS Negl. Trop. Dis. 10:e0004857. doi: 10.1371/journal.pntd.0004857

Pornsukarom, S., van Vliet, A. H. M., and Thakur, S. (2018). Whole genome sequencing analysis of multiple Salmonella serovars provides insights into phylogenetic relatedness, antimicrobial resistance, and virulence markers across humans, food animals and agriculture environmental sources. BMC Genomics. 191, 1-14. doi: 10.1186/S12864-018-5137-4

Porwollik, S., Santiviago, C. A., Cheng, P., Florea, L., Jackson, S., and McClelland, M. (2005). Differences in gene content between Salmonella enterica serovar enteritidis isolates and comparison to closely related serovars gallinarum and Dublin. J. Bacteriol. 187, 6545-6555. doi: 10.1128/JB.187.18.6545-6555. 2005

Salihu, M., Garba, B., and Isah, Y. (2015). Sokoto Journal of Veterinary Sciences Evaluation of microbial contents of table eggs at retail outlets in Sokoto metropolis. Nigeria. Sokoto J. Vet. Sci. J. Vet. Sci. 13, 22-28. doi: 10.4314/sokjvs. v13i1.4

Salihu, M. D., Magaji, A. A., Bashir, Garba, Badamasi, Saidu, Aliyu, Mamuda, Nasiru, Suleiman, et al. (2013). Bacteriological quality of raw meat displayed for sale at Sokoto, Sokoto state, Nigeria. Sci. J. Microbiol. 2, 134-139. doi: 10.14196/SJMI.V2I7.863

Sarowska, J., Futoma-Koloch, B., Jama-Kmiecik, A., Frej-Madrzak, M., Ksiazczyk, M., Bugla-Ploskonska, G., et al. (2019). Virulence factors, prevalence and potential transmission of extraintestinal pathogenic Escherichia coli isolated from different sources: Recent reports. Gut. Pathog. 11:10. doi: 10.1186/s13099019-0290-0

Sharma, C., Rokana, N., Chandra, M., Singh, B. P., Gulhane, R. D., Gill, J. P. S., et al. (2018). Antimicrobial Resistance: Its Surveillance, Impact, and Alternative Management Strategies in Dairy Animals. Front. Vet. Sci. 4:237. doi: 10.3389/ FVETS.2017.00237

Srivastava, S., Chapagain, M., and Gumbo, T. (2020). Effect of specimen processing, growth supplement, and different metabolic population on Mycobacterium tuberculosis laboratory diagnosis. PLoS One 15:e230927. doi: 10.1371/ JOURNAL.PONE.0230927

Stanaway, J. D., Parisi, A., Sarkar, K., Blacker, B. F., Reiner, R. C., Hay, S. I., et al. (2019). The global burden of non-typhoidal salmonella invasive disease: a systematic analysis for the Global Burden of Disease Study 2017. Lancet Infect. Dis. 19, 1312-1324. doi: 10.1016/S1473-3099(19)30418-9

Tasmin, R., Hasan, N. A., Grim, C. J., Grant, A., Choi, S. Y., Alam, M. S., et al. (2017). Genotypic and phenotypic characterization of multidrug resistant Salmonella Typhimurium and Salmonella Kentucky strains recovered from chicken carcasses. PLoS One 12:e176938. doi: 10.1371/journal.pone.0176938

Thomas, M., Fenske, G. J., Antony, L., Ghimire, S., Welsh, R., Ramachandran, A., et al. (2017). Whole genome sequencing-based detection of antimicrobial resistance and virulence in non-typhoidal Salmonella enterica isolated from wildlife. Gut Pathog. 9:66. doi: 10.1186/s13099-017-0213-x

Thung, T. Y., Mahyudin, N. A., Basri, D. F., Wan, Mohamed Radzi, Cw, Nakaguchi Y, Nishibuchi, M., et al. (2016). Prevalence and Antibiotic Resistance of Salmonella Enteritidis and Salmonella Typhimurium in Raw Chicken Meat at Retail Markets in Malaysia. J. Poult. Sci. 95, 1888-1893.

Thung, T. Y., Radu, S., Mahyudin, N. A., Rukayadi, Y., Zakaria, Z., Mazlan, N., et al. (2018). Prevalence, Virulence Genes and Antimicrobial Resistance Profiles of Salmonella Serovars from Retail Beef in Selangor, Malaysia. Front. Microbiol. 8:2697. doi: $10.3389 /$ fmicb.2017.02697

Touchon, M., Hoede, C., Tenaillon, O., Barbe, V., Baeriswyl, S., Bidet, P., et al. (2009). Organised genome dynamics in the Escherichia coli species results in 
highly diverse adaptive paths. PLoS Genet. 5:e1000344. doi: 10.1371/journal. pgen.1000344

Trevethan, R. (2017). Sensitivity, Specificity, and Predictive Values: Foundations, Pliabilities, and Pitfalls in Research and Practice. Front. Public Heal. 5:307. doi: 10.3389/FPUBH.2017.00307

Van, T. T. H., Yidana, Z., Smooker, P. M., and Coloe, P. J. (2020). Antibiotic use in food animals worldwide, with a focus on Africa: Pluses and minuses. J. Glob. Antimicrob. Resist. 20, 170-177. doi: 10.1016/J.JGAR.2019.07.031

Wang, X., Biswas, S., Paudyal, N., Pan, H., Li, X., Fang, W., et al. (2019). Antibiotic Resistance in Salmonella Typhimurium Isolates Recovered From the Food Chain Through National Antimicrobial Resistance Monitoring System Between 1996 and 2016. Front. Microbiol. 10:985. doi: 10.3389/FMICB.2019.00985

Wayne, P. A. (2019). CLSI. Performance Standards for Antimicrobial Susceptibility Testing Performance Standards for Antimicrobial Susceptibility Testing. 29th Edn. USA: CLSI.

Whistler, T., Sapchookul, P., McCormick, D. W., Sangwichian, O., Jorakate, P., Makprasert, S., et al. (2018). Epidemiology and antimicrobial resistance of invasive non-typhoidal Salmonellosis in rural Thailand from 2006-2014. PLoS Negl. Trop. Dis. 12:e0006718. doi: 10.1371/journal.pntd.0006718

Wu, B., -Dra, A., Pan, H., Dong, C., Jia, C., and Yue, M. (2021). Genomic Investigation of Salmonella Isolates Recovered From a Pig Slaughtering Process in Hangzhou. China. Front. Microbiol. 12:704636. doi: 10.3389/FMICB.2021. 704636

Yu, X., Zhu, H., Bo, Y., Li, Y., Zhang, Y., Liu, Y., et al. (2021). Prevalence and antimicrobial resistance of Salmonella enterica subspecies enterica serovar Enteritidis isolated from broiler chickens in Shandong Province. China, 20132018. Poult. Sci. 100:1016. doi: 10.1016/J.PSJ.2020.09.079
Zakaria, Z., Hassan, L., Sharif, Z., Ahmad, N., Ali, R. M., Husin, S. A., et al. (2020). Analysis of Salmonella enterica serovar Enteritidis isolates from chickens and chicken meat products in Malaysia using PFGE, and MLST. BMC Vet. Res. 16:393. doi: 10.1186/s12917-020-02605-y

Zhang, S., den Bakker, H. C., Li, S., Chen, J., Dinsmore, B. A., Lane, C., et al. (2019). SeqSero2: Rapid and improved salmonella serotype determination using whole-genome sequencing data. Appl. Environ. Microbiol. 85, e01746-19. doi: 10.1128/AEM.017 46-19

Conflict of Interest: The authors declare that the research was conducted in the absence of any commercial or financial relationships that could be construed as a potential conflict of interest.

Publisher's Note: All claims expressed in this article are solely those of the authors and do not necessarily represent those of their affiliated organizations, or those of the publisher, the editors and the reviewers. Any product that may be evaluated in this article, or claim that may be made by its manufacturer, is not guaranteed or endorsed by the publisher.

Copyright (c) 2021 Zakaria, Hassan, Ahmad, Husin, Ali, Sharif, Sohaimi and Garba. This is an open-access article distributed under the terms of the Creative Commons Attribution License (CC BY). The use, distribution or reproduction in other forums is permitted, provided the original author(s) and the copyright owner(s) are credited and that the original publication in this journal is cited, in accordance with accepted academic practice. No use, distribution or reproduction is permitted which does not comply with these terms. 